\title{
The challenging diagnosis of intravascular large B-cell lymphoma
}

\author{
Filipa Coroado Ferreira, ${ }^{1}$ Tiago Rabadão, ${ }^{1}$ Leonor Naia, ${ }^{1}$ Mariana Teixeira, ${ }^{1}$ Marcelo Aveiro, ${ }^{1}$ Gisela Ferreira, ${ }^{2}$ \\ Diana Aguiar ${ }^{1}$
}

${ }^{1}$ Internal Medicine Department; and ${ }^{2}$ Hematology Department, Centro Hospitalar do Baixo Vouga, Aveiro, Portugal

\begin{abstract}
Livedo reticularis (LR) is a net-like cyanotic skin pattern resulting from cutaneous blood flow disturbance associated with several medical conditions. The authors present a case of a 77-year-old woman with a history of asthenia over one year, admitted to the hospital with sepsis by pneumonia. During her hospitalization, she developed a livedoid rash, and skin biopsy revealed infiltrates of atypical mononuclear cells causing capillary lumen obstruction. Intravascular large B-cell lymphoma was suspected and confirmed by bone marrow study. This case highlights the importance of being aware of different medical conditions associated with LR and the atypical presentation of a lymphoproliferative disorder.
\end{abstract}

\section{Case Report}

A 77-year-old woman with asthenia over one year presented to the emergency department with a produc-

Correspondence: Ana Filipa Coroado da Silva Ferreira, Internal Medicine Department, Centro Hospitalar do Baixo Vouga, Aveiro, Av. Artur Ravara, 3810-501 Aveiro, Portugal. Tel.: 234.378.300. E-mail: afilipa.csferreira@gmail.com

Key words: Livedo reticularis; lymphoproliferative disorder; intravascular lymphoma.

Acknowledgments: the authors would like to express my deep gratitude to Dr. Joana Pires for her guidance and enthusiastic encouragement for this case report. My grateful thanks are also extended to Dr. Domingos Oliveira and Helena Garcia for biopsies' images.

Contributions: FF, lead author, literature review and text editing; TR, literature review and text editing; LN, literature review and text editing; MT, text editing and revising it critically for important intellectual content; MA, drafting the article and text editing; GF, text editing and validation of scientific content; DA, text editing, validation of scientific content, final approval of the version to be published.

Conflict of interests: the authors declare no potential conflict of interests.

Ethics approval: compliance with ethical standards.

Received for publication: 30 October 2020.

Accepted for publication: 9 November 2020.

This work is licensed under a Creative Commons Attribution NonCommercial 4.0 License (CC BY-NC 4.0).

${ }^{\circ}$ Copyright: the Author(s), 2021

Licensee PAGEPress, Italy

Italian Journal of Medicine 2021; 15:64-66

doi:10.4081/itjm.2021.1413 tive cough, purulent sputum and dyspnoea for small efforts. Clinically she was hypotensive, unresponsive to fluids, tachypneic, feverish and pulmonary auscultation revealed basal crackles and wheezing. The laboratory tests revealed elevated inflammatory markers, acute kidney injury (creatinine $1.59 \mathrm{mg} / \mathrm{dL}$ ), normochromic normocytic anemia (hemoglobin 8.4 $\mathrm{g} / \mathrm{dL}$ ), and detection of Streptococcus pneumoniae in sputum (positive polymerase chain reaction). A thoracic radiograph revealed an infiltrate in the inferior right lobe. The diagnosis of a septic shock by pneumonia was established, and she was admitted to the Intensive care unit. After improvement, the patient was transferred to the internal medicine ward. Revisiting clinical information, the patient had worsening asthenia in the last 2 months, presented long-time anemia, dysphagia, loss of appetite, and unquantified weight loss.

Several laboratory tests were ordered. Results included complete blood count with pancytopenia, erythrocyte sedimentation rate of $36 \mathrm{~mm} / \mathrm{hr}$, lactate dehydrogenase $>650 \mathrm{U} / \mathrm{L}$, for an upper limit value of $246 \mathrm{U} / \mathrm{L}$, and ferritin $2145 \mathrm{ng} / \mathrm{dL}$. Further investigations revealed positive antinuclear autoantibodies (title $1 / 640$, with fine speckled pattern), hypocomplementemia, and polyclonal hyperglobulinemia with an elevated $\beta 2$-microglobulin. Bacteriological exams were unremarkable (blood cultures and serological tests for Cytomegalovirus, Epstein-Barr virus, Rickettsia, Coxiella, and Borrelia).

Thoracic-abdominopelvic CT scan revealed no other findings besides pulmonary edema suggestive of congestive heart failure, confirmed by transthoracic echocardiogram. Right pleural effusion was interpreted in the context of right heart failure and therefore not addressed; instead, it was treated with a diuretic that produced a slight improvement.

The patient evolved with neurologic impairment 
and livedoid rash in the back, trunk (Figure 1), and proximal legs, with no alteration on the external temperature. A skin biopsy, bone marrow aspiration, and biopsy, with the evaluation of cytology, flow cytometry, and karyotype, were performed. Unfortunately, the patient died before the results were known.

Skin biopsy revealed infiltrates of mononuclear cells with marked atypia in the hypodermis' capillary lumen leading to vessel obstruction without necrosis (Figure 2). Those cells were CD20 positive (Figure 3) and CD3 negative. These findings were suggestive of intravascular large B-cell lymphoma.

The medullogram test revealed pleomorphic cells of variable size with a very irregular nucleus, sometimes very lobulated, with lax chromatin and one or two nucleoli, accounting for $41 \%$ of all nucleated cells. Large amounts of iron were present at the eritron, and no visible ring sideroblasts. Flow cytometry of medullar aspirate revealed the presence of $33 \%$ of large clonal lambda B cells with a phenotype suggestive of diffuse large B cell lymphoma (CD10 positive), T and NK cells with normal phenotype. The karyotype revealed complex and multiple clonal numerical and structural chromosomal alterations associated with a poor prognosis. Bone marrow biopsy was consistent with involvement by diffuse large B cell lymphoma.

\section{Discussion}

Livedo reticularis (LR) is an ischemic dermopathy characterized by a reticular mottling surrounding a pallor central core of skin. This phenomenon is explained by reduced blood flow to the skin, increasing the proportion of deoxygenated hemoglobin. It is a common finding in several medical conditions and usually benign; however, it can be associated with complex clinical settings such as infections, autoimmune or hematologic conditions, drugs, and malignancies, namely in lymphoproliferative disorders. ${ }^{1}$

In the presented case, sepsis could explain the LR; however clinical improvement and a more detailed clinical history raised the suspicion of other etiologies. Laboratory findings suggested the presence of lymphoproliferative disorder, including anemia with rapid evolution to pancytopenia, elevated $\beta 2$-microglobulin, lactate dehydrogenase, and erythrocyte sedimentation rate. Antiphospholipid syndrome and other autoimmune disorders were excluded. Skin biopsy revealed histopathological signs of intravascular large B-cell lymphoma, and the medullar study confirmed diffuse large B cell lymphoma with a poor prognosis karyotype.

Intravascular large B-cell lymphoma is a rare subtype of diffuse large B-cell lymphoma, characterized by the proliferation of clonal B-cells within small blood vessel lumen, without an obvious extravascular tumor

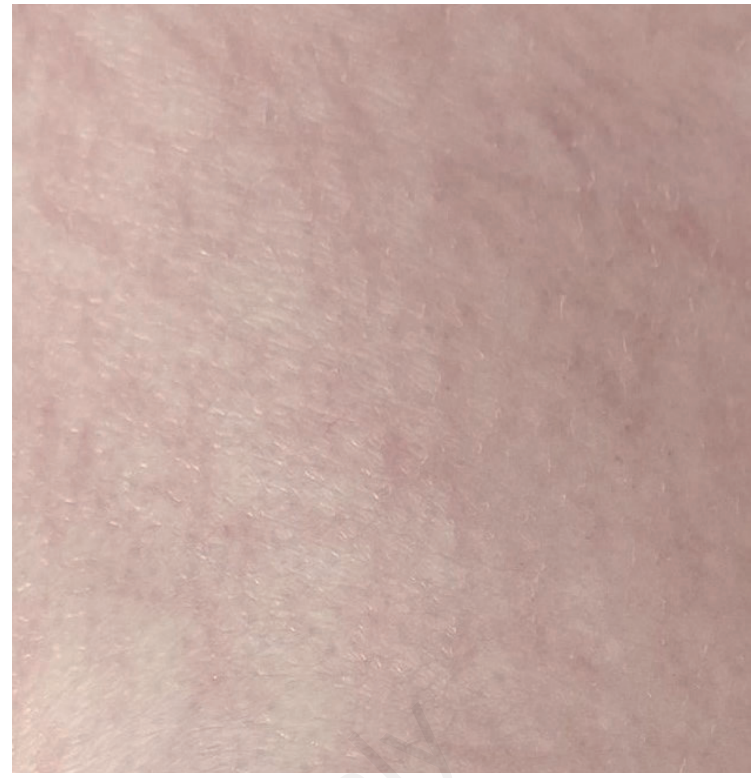

Figure 1. Livedoid rash in patient's abdomen.

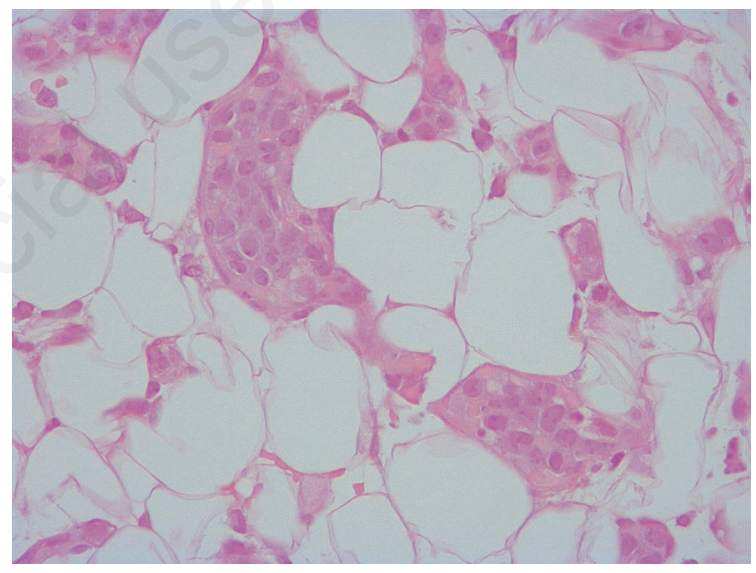

Figure 2. Obstructed capillaries by atypical mononucleated cells on hematoxylin-eosin staining $(400 \times$ magnification).

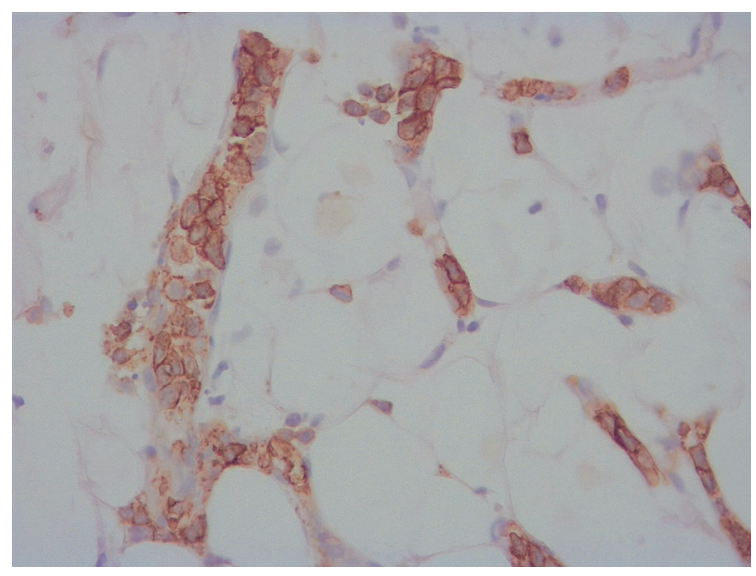

Figure 3. Obstructed capillaries by atypical CD20 positive mononucleated cells $(400 \times$ magnification $)$. 
mass. It presents with various symptoms that include constitutional B symptoms, rapidly progressive neurologic impairment, and skin lesions mimicking inflammatory skin conditions. ${ }^{2}$ The diagnosis is established by skin biopsy, which reveals large lymphoma cells within the blood vessels, and treatment includes systemic therapy and therapy directed at the central nervous system. ${ }^{3,4}$ The overall mortality is associated with the prognosis of the lymphoproliferative disorder itself, but when the disease is restricted to the skin, the prognosis seems to be more favorable. ${ }^{5}$ Early diagnosis and treatment could reduce mortality and improve prognosis, although it may not translate into a different outcome given the poor prognosis of this case.

\section{Conclusions}

The diagnosis of lymphoproliferative disorder should be considered in patients with constitutional symptoms who develop vasculitis-like cutaneous signs/livedoid rash after excluding other more common causes. Livedoid rash is not a common sign of the presence of a lymphoproliferative disorder. The authors want to emphasize the importance of awareness of the differential diagnosis of LR and atypical presentation of lymphoproliferative disorders. We believe that early recognition may help establish an early diagnosis and improve clinical outcome, although it may not translate into a different outcome.

\section{References}

1. Dean MS. Livedo reticularis and related disorders. Curr Treat Options Cardiovasc Med 2011;13:179-91.

2. Röglin J, Böer A. Skin manifestations of intravascular lymphoma mimic inflammatory diseases of the skin. $\mathrm{Br}$ J Dermatol 2007;157:16-25.

3. Ponzoni M, Campo E, Nakamura S. Intravascular large B-cell lymphoma: a chameleon with multiple faces and many masks. Blood 2018;132:1561-7.

4. Ong YC, Kao HW, Chuang WY, et al. Intravascular large B-cell lymphoma: a case series and review of literatures. Biomed J 2020;S2319-4170(20)30041-X.

5. Charifa A, Paulson N, Levy L, et al. Intravascular large B-Cell lymphoma: clinical and histopathologic findings. Yale J Biol Med 2020;93:35-40. 